\title{
BMJ Open Strength training as a supplemental therapy for androgen deficiency of the aging male (ADAM): study protocol for a three-arm clinical trial
}

\author{
Michal Kralik, ${ }^{1}$ Jan Cvecka, ${ }^{1}$ Gabriel Buzgo, ${ }^{1}$ Matus Putala, ${ }^{1}$ \\ Barbara Ukropcova, ${ }^{1,2,3}$ Jozef Ukropec, ${ }^{4}$ Zdenko Killinger, ${ }^{5}$ Juraj Payer, ${ }^{5}$ \\ Boris Kollarik, ${ }^{6}$ Peter Bujdak, ${ }^{7}$ Truls Raastad, ${ }^{8}$ Milan Sedliak ${ }^{1}$
}

To cite: Kralik M, Cvecka J, Buzgo G, et al. Strength training as a supplemental therapy for androgen deficiency of the aging male (ADAM): study protocol for a threearm clinical trial. BMJ Open 2019;9:e025991. doi:10.1136/ bmjopen-2018-025991

- Prepublication history and additional material for this paper are available online. To view these files, please visit the journal online (http://dx.doi org/10.1136/bmjopen-2018025991).

Received 12 August 2018 Revised 30 May 2019 Accepted 03 July 2019
Check for updates

(C) Author(s) (or their employer(s)) 2019. Re-use permitted under CC BY-NC. No commercial re-use. See rights and permissions. Published by BMJ.

For numbered affiliations see end of article.

Correspondence to Dr Milan Sedliak; milan.sedliak@uniba.sk

\section{ABSTRACT}

Introduction Androgen deficiency of the ageing male is a clinical syndrome resulting from the low production of androgens (testosterone levels $<6.9 \mathrm{nmol} / \mathrm{L}$ ) with symptoms including decline in lean mass, muscle strength, increases in body mass and overall fat mass. The aim of the study is to examine the effect of a 12 week strength training intervention on body composition, physical function, muscle cellular and molecular and selected biochemical markers of metabolic health in hypogonadal patients.

Methods and analysis The study is three-group controlled 12-week experiment to assess the effect of strength training on hypogonadal patients with testosterone replacement therapy and newly diagnosed males without testosterone replacement therapy. Age matched healthy eugonadal males are also engaged in strength training. Lean mass is used to determine sample size indicating, that 22 subjects per group will be sufficient to detect intervention related changes at the power of 0.90 . All outcomes are collected before the intervention (pre-intervention assessments) and after the intervention (post-intervention assessments). Clinical outcomes are body composition (lean mass, fat mass and total body mass) measured by dual-energy X-ray absorptiometry, physical functioning assessed by physical tests and psychosocial functioning. The most important haematological and biochemical parameters included are glucose, total cholesterol, low-density lipoprotein cholesterol, high-density lipoprotein cholesterol, testosterone, luteinizing hormone, follicle-stimulating hormone, sexhormone-binding globulin, insulin and prostate-specific antigen. Muscle cellular and molecular outcomes are muscle fibre size and regulators of muscle fibre size. Muscle cellular outcomes are measured from muscle biopsies obtained from musculus vastus lateralis Ethics and dissemination This trial is approved by Ethics Committee of the University Hospital in Bratislava, Slovakia, (ref. trial number: 127/2017) and all subjects will be fully informed on the rationale, risks and benefits of the study and sign the written informed consent prior to entering the study. Results will be published in peerreviewed journals and presented in scientific conferences. Trial registration number NCT03282682
Strengths and limitations of this study

- To the best of our knowledge this trial represents the first study in hypogonadal males focusing on possible physiological and metabolic mechanisms of strength training at circulating, cellular and molecular level.

- Wide spectrum of clinical outcomes with high-standard methods of assessments (dual-energy X-ray absorptiometry, muscle biopsies).

- The major limitation of this trial is small sample size, caused by limited number of detected patients.

- Another limitation is that the participants will be asked to not change their habitual dietary intake during the intervention, but the actual intake is not monitored.

\section{INTRODUCTION}

Testosterone is one of the most potent naturally secreted androgenic-anabolic hormone, and its biological effects include, among others, promotion of skeletal muscle growth. ${ }^{1}$ Testosterone stimulates protein synthesis, inhibits protein degradation and these effects account for the promotion of muscle hypertrophy by testosterone. ${ }^{2}$ Ageing beyond 35 to 40 years is associated with a decline of $1 \%$ to $3 \%$ per year in circulating testosterone concentration $(1.6 \%$ in total and $2 \%$ to $3 \%$ in bioavailable testosterone) in men. This reduction can eventually lead to very low resting concentrations of circulating testosterone, a condition that has been termed andropause. ${ }^{3-6}$ Although the lower limit of normal total testosterone is not clearly defined, American Association of Clinical Endocrinologists suggests $6.9 \mathrm{nmol} / \mathrm{L}$ as lower limit of normal testosterone levels, other societies suggest 8 $\mathrm{nmol} / \mathrm{L}$ and even up to $10 \mathrm{nmol} / \mathrm{L}^{7}$ as a limit below which patients can be considered as hypogonadal. The Endocrine Society defines 


\section{Primary hypogonadism}

Congenital anorchidism, cryptorchidism, mumps orchitis

Genetic and developmental conditions:

Klinefelter syndrome, androgen receptor

and enzyme

Defects, Sertoli cell only syndrome

Radiation treatment/chemotherapy

Testicular trauma autoimmune syndromes

(anti-Leydig cell disorders)

\section{Secondary hypogonadism}

Genetic conditions:

Kallmann's syndrome, Prader-Willi

syndrome

Pituitary tumours, granulomas,

abscesses Hyperprolactinaemia

cranial trauma Radiation treatment

Various medications
Mixed (primary and secondary) hypogonadism*

Alcohol abuse ageing chronic infections (HIV) corticosteroid treatment Haemochromatosis systemic disease (liver failure, uraemia, sickle-cell disease) *Mixed hypogonadism is often included within the secondary hypogonadism category. male hypogonadism as a clinical syndrome resulting from failure of the testis to produce physiological levels of testosterone (androgen deficiency) and normal number of spermatozoa. Hypogonadism (primary, secondary or mixed) is caused by disruption of one or more levels of the hypothalamic-pituitary-gonadal axis. ${ }^{8}$ All the causes of male hypogonadism can be found in table $1 .^{9-11}$ Due to complexity of the diagnosis of hypogonadism, there are several alternative names for male hypogonadism, but for a purpose of this trial, the term ADAM was chosen.

\section{Symptoms of hypogonadism}

Total testosterone is a reliable marker for the initial screening of men presenting symptoms of hypogonadism, ${ }^{1-14}$ (figure 1), but for better understanding of the ADAM syndrome it is sometimes required to analyse also free or bioavailable testosterone. ${ }^{15}$ Most testosterone circulates tightly bound to sex hormone-binding globulin (SHBG) or weakly bound to albumin. A minor amount

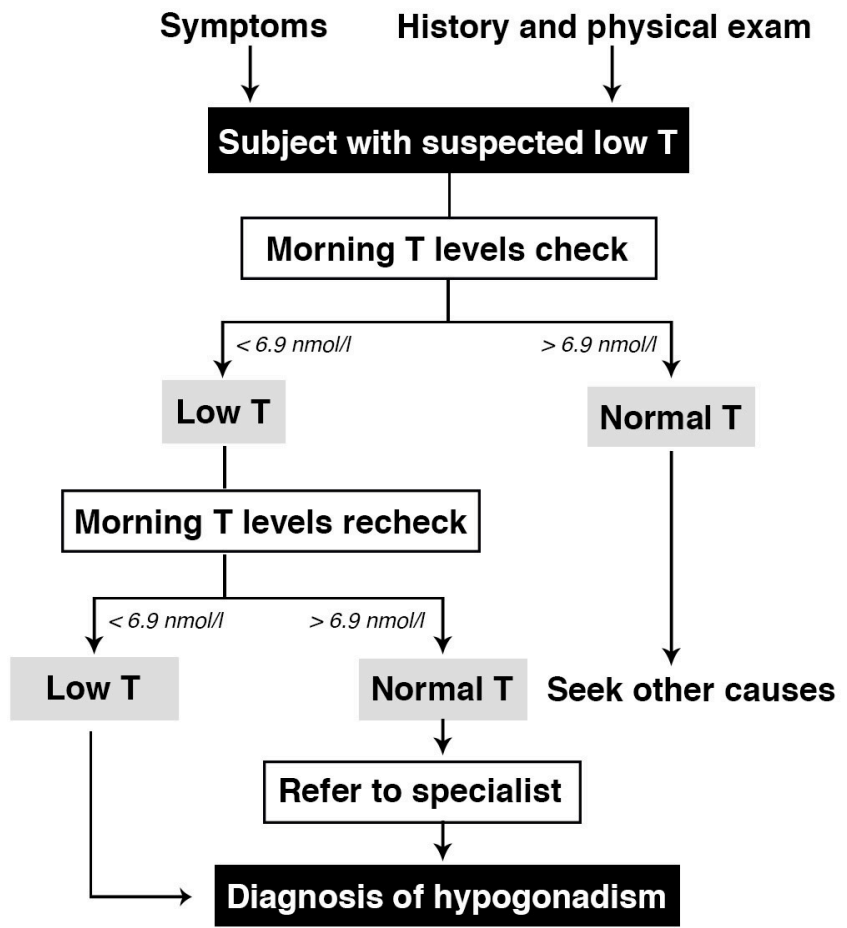

Figure 1 An algorithm for the diagnosis of hypogonadism (T - total testosterone) according to ${ }^{71213}$ (adapted from ${ }^{3}$ ). circulates as free testosterone, and it is believed that this is the metabolically active fraction. Therefore, measurements of free testosterone is important in the diagnosis of disorders of androgen deficiency in men. ${ }^{16}$

Symptoms of male hypogonadism include decline in lean mass (LM), muscle strength, adiposity, libido and erectile dysfunction, depressed mood, decreased energy or vitality, increased fatigue, osteoporosis or low bone mass, increases in body mass and overall fat mass. ${ }^{817}$ Studies of hypogonadal men shows, that there are increases in body mass and overall body fat mass as well as decreases in LM with declining androgen levels. ${ }^{17}$ Androgens also have a direct impact on bone mineral density since testosterone and oestrogens both play a vital role in bone health and low testosterone levels can cause an increase in osteoclast induced bone resorption. ${ }^{18}$ Other sources also state direct correlation between low testosterone levels and increased risk of aortic atherosclerosis independent of age, increased body mass index (BMI), total cholesterol or diabetes. ${ }^{19}$

These symptoms may affect men earlier in life, already in their late third decade of life. ${ }^{20}$ If untreated, chronic lower than normal testosterone level dramatically increases risk of many diseases later in life. Studies have suggested a link between hypogonadism and cardiovascular disease, which is not surprising given the relationship with hypogonadism and the metabolic syndrome. ${ }^{1021}$ Testosterone is a hormone regulating several pathways affecting many other syndromes, for example locomotive syndrome. ${ }^{22}$ There is a likely causal relationship between low androgen levels and ageing, as well as its association with increased risk and the occurrence of cardiovascular events and progression of cardiovascular diseases. ${ }^{23}$ On a metabolic level, men with lower androgen levels have demonstrated higher glucose and insulin levels, higher rates of obesity and increased incidence of type 2 diabetes and other diseases. ${ }^{24-28}$

\section{Testosterone replacement therapy}

For decades hypogonadism, has been treated by testosterone replacement therapy (TRT) lifelong, as this helps to prevent some of the adverse health effects. ${ }^{29-31}$ Restoration of testosterone levels to the normal range improves libido, sexual function and mood, reduces fat body mass, 
increases lean body mass and improves bone mineral density. $^{3}$

Among the published trials on the role of testosterone in older men, not all report increased muscle strength with testosterone replacement therapy. The studies reporting significant strength gains were performed in hypogonadal subjects and employed a higher dose of testosterone for a longer duration. ${ }^{32}$ Nair $e t a l^{33}$ described in their report treatment of a group of hypogonadal men with a transdermal testosterone at a dose of $35 \mathrm{mg}$ / week for 24 months and found no increase in strength. However, $35 \mathrm{mg} /$ week is less than a replacement dose and resulted in only a $30 \%$ increase in the circulating testosterone concentration. Some other studies ${ }^{34-37}$ also report small or no increases in muscle strength with TRT. Maintenance of the musculoskeletal system by increased bone density will contribute to increased physical fitness, reflected by increased strength and endurance, ${ }^{38}$ and the treatment outcome is strongly influenced by age and training. ${ }^{38}$ Lašaite $e t a l^{39}$ observed that 2 year testosterone replacement therapy in young and middle-aged hypogonadal men had beneficial effect on cognitive functioning (improved attention and visual scanning ability, executive function and psychomotor speed), but not on emotional state and quality of life. Hildreth $e t a t^{40}$ found that TRT improved body composition, but it had no effect on functional performance. Testosterone replacement can improve lipid and insulin metabolism, resulting in changes of body composition, such as decreasing fat depots and growth of muscle fibres can also be observed. ${ }^{39}$ Permpongkosol $e t a t^{41}$ in their work from 2016 found that 8 year treatment of long-acting testosterone undecanoate did not improve all obesity parameters.

It is still not clear how testosterone effects cognitive function in adult men, but testosterone may exert its action through androgen receptors in the brain and has been shown effect on serotonin, dopamine, acetylcholine and calcium signalling. ${ }^{42}$ Barrett-Connor $e t a t^{33}$ found correlation between higher bioavailable testosterone and better scores on 2 of 12 cognitive function tests. Higher total or bioavailable testosterone levels tended to be associated with better performance on tests with verbal memory and mental control. Testosterone enhanced cerebral perfusion in hypogonadal men and that perfusion takes place specifically in Brodmann areas 8 and 24, regions of the brain that are concerned with: strategic planning, higher motor action, cognitive behaviours, emotional behaviour, generalised emotional reaction, wakefulness and memory. ${ }^{44}$ Hypogonadal men have lower scores in tests of memory, visuospatial function, with a faster decline in visual memory. ${ }^{27}$ McIntyre $e t a t^{45}$ found, that middle-aged males with depressions did have a reduction in bioavailable testosterone.

\section{Risks associated with testosterone replacement therapy}

Testosterone treatment is contraindicated in subjects with breast cancer or benign prostate hyperplasia, lower urinary tract symptoms and if risks of treatment is perceived to be high by many physicians. ${ }^{3}$ The risk of prostate cancer with TRT is still unclear. Only intramuscular treatment found slight increase in prostate-specific antigen (PSA) levels. ${ }^{46}$ Loeb et $a l^{47}$ found that TRT remained significantly associated with more favourable-risk prostate cancer and lower risk of aggressive prostate cancer. But other studies and meta-analysis found TRT as a safe urological approach to treat hypogonadism. ${ }^{4-50}$ Other risks of TRT in men include fluid retention, mood fluctuations, gynaecomastia, worsening of sleep apnoea, polycythaemia, elevation of PSA. ${ }^{350}$ Bhasin et $a \tilde{l}^{1}$ found higher incidence of adverse effects (included haematocrit greater than 54\%, leg oedema with shortness of breath, urinary retention and prostate cancer) in treating older men with the very high doses of totaltestosterone $(\mathrm{T})$ compared with young males. Rhoden and Morgentaler ${ }^{52}$ have reviewed the adverse effects and recommend the long-term monitoring of the above-mentioned parameters. Potential adverse events not related to hormones include pain at injection site and local skin irritation.

\section{The effects of strength training}

Much research has been conducted on the effect of strength protocols on muscle mass and muscle strength which incorporate large muscle groups at intensities around $70 \%$ to $80 \%$ of 1RM (one repetition maximum), volumes from two to three sets of 10 to 12 repetition and rest periods of short to medium duration (60 to $90 \mathrm{~s}) .5354$ Beneficial effects of exercise, especially resistance training have been clearly shown with regards to the quality of life, fatigue, muscle strength, muscular endurance and functions and body composition in elderly men with prostate cancer receiving androgen-deprivation therapy, thus being in a chronically low testosterone condition. ${ }^{55} 56$ Clearly, resting levels of testosterone and other androgens but not their acute elevations due to exercise have also impact on muscle hypertrophy as suggested by a recent review article. ${ }^{57}$ As for exercise interventions with ADAM patients, the scientific evidence is very limited but promising. Schwarz and Willix ${ }^{23}$ found positive outcomes on coronary risk factors such as glucose intolerance and hyperlipidaemia when TRT was combined with endurance exercise. To our knowledge, only Hildreth $e t a l^{40}$ have used resistance training and found benefits of both resistance exercise with TRT as well as without TRT in hypogonadal males. After intervention, there were no significant differences between combination of resistance exercises with TRT or with placebo in improvements in muscle function or strength in the two exercise groups. However, adding TRT resulted in greater improvements in decrease of fat mass and increase of fat-free mass. In the TRT but no exercise condition, patients did not improve muscle function but decreased fat mass, increased fat-free mass and upper body strength. Importantly, TRT plus progressive resistance training produced greater improvements in body composition than either intervention alone.

Glintborg et $a p^{\tilde{8}}$ studied effects of TRT and/or strength training (ST) on cardiovascular risk in hypogonadal 
males for 6 months. This double-blinded, placebo-controlled study found that only ST + placebo significantly decreased soluble cluster of differentiation 36 (sCD36) levels. Only placebo group did not decrease fat mass during this period. Compared with TRT, 6 months of strength training reduced sCD36 levels suggesting decreased cardiovascular risk, possibly due to a reduction in central fat mass.

In a pilot randomised controlled trial by Cho and colleagues $^{59}$ when hypogonadal males were treated with combination of exercise and TRT, significantly better results in serum testosterone levels and symptoms of hypogonadism compared with TRT alone after 12 weeks of intervention were found. The levels of testosterone were significantly higher in the combination group ( $\mathrm{p}=0.01$ ) In addition, these improvements were well-maintained in the combination group with continuous exercise even after cessation of TRT. After 20 weeks of intervention the group which used TRT and strength training kept the testosterone levels significantly higher $(p=0.01)$ compared with the group with TRT only. Consequently, it seems that exercise can augment the durability of response to TRT and it may be the solution to shorten the treatment duration with a lower risk from testosterone therapy. ${ }^{59}$ There are some very promising results showing a great potential of exercise in hypogonadal patients. However, the above-mentioned studies did not focus on possible physiological and metabolic mechanisms responsible for the positive effects of resistance training at circulating, cellular and molecular level. Up to date, there are no studies investigating the effects of strength training on the regulation of muscle mass and neuromuscular function at a cellular level in hypogonadal male patients.

\section{Aims}

The overall aim of the trial is to examine the effect of a 12-week strength training programme with and without TRT on body composition, physical function, selected biochemical markers of metabolic health, histological and molecular parameters and the quality of life of patients with ADAM.

\section{Study design}

The study is a clinical trial with three arms comparing the effect of strength training with testosterone replacement therapy (ST + TRT), strength training alone (ST) on hypogonadal males and on a control group of healthy eugonadal males (HM), also engaged in strength training for 12 weeks (figure 2).

\section{Trial status}

At the time of the first submission of the protocol, the trial was in the phase of participant recruitment. The recruitment began in February 2017 and the last part of data collection is expected to end in August 2019.

\section{Participants}

Subjects will be included from urological units at Department of Urology, University Hospital-Petrzalka, Bratislava,

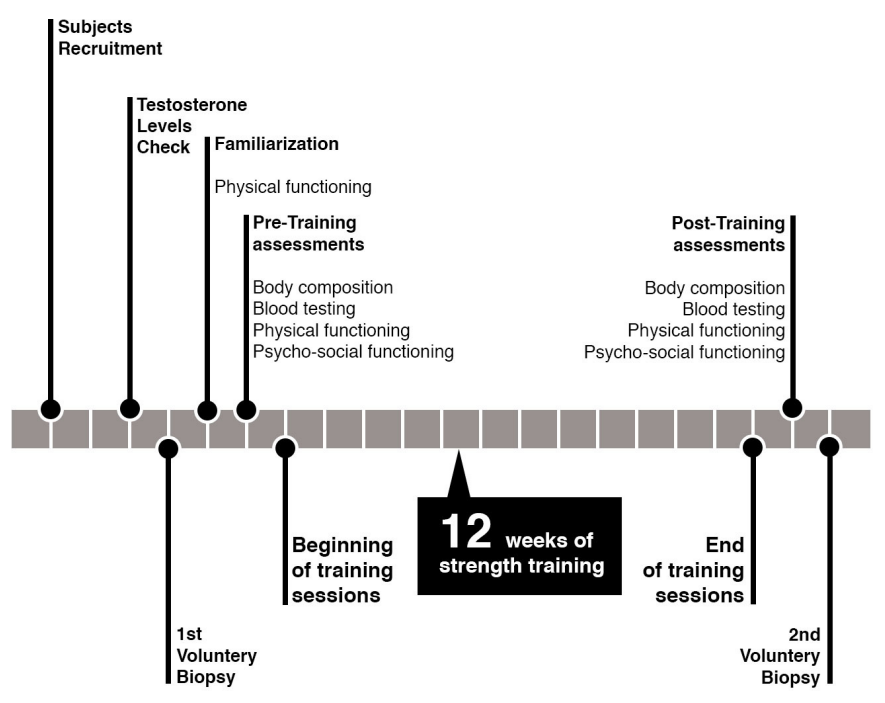

Figure 2 Timeline of the ADAM study.

Slovakia; Department of Urology, Faculty of Medicine, Comenius University, Bratislava, Slovakia and $5^{\text {th }}$ Department of Internal Medicine, Faculty of Medicine, Comenius University, Bratislava, Slovakia. The study will involve in total 66 male participants divided into three groups $(\mathrm{n}=66)$ : group 1 , males with hypogonadism who are undergoing TRT ( $\mathrm{n}=22)$; group 2 , newly diagnosed males with hypogonadism without testosterone replacement therapy (NON-TRT) (n=22); group 3, healthy eugonadal men (HM) $(n=22)$. The participants from all groups engaged in strength training. The volunteers are screened for testosterone levels before the start of the participation by the specialist.

The most important inclusion criteria for participation in the study from the patient population are age 40 to 60 years old, subjects with hypogonadism on TRT or newly diagnosed patients of hypogonadism. The hypogonadal patients fulfilling the criteria for study participation will be verified for low testosterone before entering the study. The same verification will take place at the end of the study. The most important exclusion criteria include regular strength training, conditions that are medical contraindications and prostate cancer or abnormal serum PSA levels without adverse histological examination. All inclusion and exclusion criteria are listed in online supplementary additional file 1 . In addition to written information, eligible subjects will be verbally informed about the study by their responsible urologist and the study officials before participation. TRT provided to patients is intramuscular injection of testosterone undecanoate (TU) at a dose of $1000 \mathrm{mg}$ repeated every 12 weeks. Testosterone undecanoate (Nebido) is the only injectable form of testosterone used at the institutes of collaborating physicians of the study. According to our knowledge, this form of $\mathrm{T}$ at dose of $1000 \mathrm{mg}$ is the most stable of all available preparations for 3 months' period, which is considered a standard treatment in Slovakia. Shorter-acting forms may cause more pronounced fluctuations in 24 hours circulating levels of testosterone. The participants will be asked 
Table 2 Dynamic warm-up

\begin{tabular}{ll}
\hline Dynamic warm-up exercises & Number of repetitions \\
\hline Walking low skip & Eight times each leg \\
Walking high knee skip & Eight times each leg \\
Walking knee to chest & Eight times each leg \\
Walking hamstring stretch & Six times each leg \\
Walking lunge & Six times each leg \\
Standing lateral lunge & Six times each leg \\
\hline Egyptian mobility exercise & Six times each arm \\
External rotation exercise & Six times each arm \\
\hline Hip hinge exercise & Eight times \\
Air squat & Eight times \\
\hline
\end{tabular}

to not change their habitual dietary intake and physical activity patterns. Participants will be asked to continue in physical activities as before, but any kind of regular physical activity, especially strength training or any other kind of weight training during the intervention will be also prohibited. The exclusion criteria reject any participant, who performed any kind of regular strength training 1 year prior to study.

\section{Strength training intervention}

The strength training protocol will be a modified strength exercise programme from Segal et at ${ }^{60}$ which was used in similar group of patients. The participants will perform 24 training sessions of strength training protocol with the frequency of two training sessions per week for 12 weeks. There will be at least 48 hours rest period between two subsequent training sessions (Monday and Thursday). The intervention will take place at the Faculty of Physical Education and Sport, Comenius University in Bratislava, Slovakia. All training sessions will be supervised and guided by professionals with university degree in sports training to ensure safety, correct technique and progression in training load, with a maximum of three participants per one trainer. The participants will be familiarised with the equipment and exercise technique 1 week before the start of the intervention. The technique corrections will be possible during the whole intervention if needed. Ten repetition maximum (RM) and 12RM diagnostic test for all exercises will be conducted during the first week of training intervention. Each training session will include a 5 min dynamic warm-up, consist of 10 exercises for approximately $30 \mathrm{~s}$ of each, and exercises will be focused on main muscle groups (table 2 ).

The strength protocol exercises will be performed with free weights and on machines. The training programme consist of six exercises for upper and lower body at an intensity of $60 \%$ to $80 \%$ ( 8 to $12 \mathrm{RM}$ : the load that induces technique failure in eight or twelve repetitions) of one-repetition maximum and takes approximately $60 \mathrm{~min}$. The inability to perform full repetition will be assessed by a supervisor or by participants' feedback. The participants will be instructed to perform a concentric action for $2 \mathrm{~s}$ and immediately after an eccentric action also for $2 \mathrm{~s}$. There will be $90 \mathrm{~s}$ rest period after each set. The same duration rest period will be between all of the exercises. The rest periods will be controlled by timer (The miniMAX, Gymboss, USA). The load will be added, if participant can complete prescribed number of repetition in each set of the exercise. More detailed strength training protocol can be seen in table 3. During the first 3 weeks of the intervention, there will be one set in the beginning with light weight to focus on safety and technique. After that three more sets with weight close to $60 \%$ to $80 \%$ of $1 \mathrm{RM}$ will follow. After first 3 weeks, the number of sets will be increased to four.

The exercises performed during every session will be: leg press, split squat, bench press. The exercises alternating through the week are knee extension with leg curl, seated row with seated pull down and incline dumbbell bench press (training equipment provided by $\mathrm{KOHI}$ Leopoldov, Slovakia, and Technogym, Italy). Since unilateral exercises (eg, one leg squats) develop similar magnitude of muscle activity with producing less load on the spine, thus they are safer, ${ }^{61}$ the split squats are chosen instead of regular squats. Prescribed exercises in the strength training protocol for every training sessions can be found in table 4 . Each session will be supervised by at least two professionals, who received strength training programme and record every repetition and set made in each session in an individual training plan. At the start of each session, the trainers will ask participants if they experienced any adverse events since the last session and record reported events. All adverse events during the training session will be written down into paper spread sheet and processed afterwards. Each training session will be monitored with an attendance list, with minimum

Table 3 Strength training protocol

\begin{tabular}{|c|c|c|c|c|c|c|}
\hline Week & Number of exercises & Number of sets & Number of repetitions & Resistance & Rest period & Tempo \\
\hline 1-3 week & $3+3$ (UB, LB) & 3 & $10-12$ & 10-12RM & $90 \mathrm{~s}$ & $2: 0: 2: 1$ \\
\hline 4-6 week & $3+3$ (UB, LB) & 4 & $10-12$ & 10-12RM & $90 \mathrm{~s}$ & $2: 0: 2: 1$ \\
\hline 10-12 week & $3+3$ (UB, LB) & 4 & $6-8$ & 6-8RM & $90 \mathrm{~s}$ & $2: 0: 2: 1$ \\
\hline
\end{tabular}

LB, lower body; RM, repetition maximum; Tempo, duration in seconds during the repetition - $2 \mathrm{~s}$ (eccentric), $0 \mathrm{~s}$ (end range of the motion), $2 \mathrm{~s}$ (concentric), $1 \mathrm{~s}$ (rest between repetitions in the starting position); UB, upper body. 


\begin{tabular}{llll}
\multicolumn{2}{l}{$\begin{array}{l}\text { Table } 4 \\
\text { resistance }\end{array}$} & Training sessions, type of exercises and type of \\
\hline $\begin{array}{l}\text { First training } \\
\text { session }\end{array}$ & $\begin{array}{l}\text { Type of } \\
\text { resistance }\end{array}$ & $\begin{array}{l}\text { Second } \\
\text { training } \\
\text { session }\end{array}$ & $\begin{array}{l}\text { Type of } \\
\text { resistance }\end{array}$ \\
\hline $\begin{array}{l}\text { Split squat } \\
\text { Bench press }\end{array}$ & Dumbbells & Bench press & Barbell \\
Leg press & Machine & Split squat & Dumbbells \\
Seated row & Machine & Leg press & Machine \\
Leg curl & Machine & Pull down & Machine \\
Lateral raise & Dumbbells & Knee & Machine \\
& & extension & \\
\hline
\end{tabular}

$85 \%$ attendance during the study. Each session will be marked as successfully completed when at least $80 \%$ from the total volume and intensity of the training protocol planned for the particular training session is performed. If a participant will be unable to perform any of the exercises or sets, this will be recorded into a prepared training plan and the situation will be managed during the first week during familiarisation with the training protocol. The appropriate alternative exercise will be considered depending on the restriction or participant's limitation.

\section{Clinical outcomes}

Clinical outcomes will be collected 1 week before the intervention (pre-intervention assessments) and 1 week after the intervention (post-training assessments). All outcomes, specific variables and assessments in each testing are listed in online supplementary additional file 2. All participants will be tested at the same time of the day, and asked to avoid caffeinated and alcohol beverages before the assessments.

\section{Familiarisation}

To secure validity of the physical tests, all subjects undergo a session of familiarisation 7 days prior to the intervention assessments. All sessions are performed based on the same guidelines, but after the familiarisation session the load of each resistance exercise will be adjusted to match the expected maximum.

\section{Primary outcome measure lean mass}

The primary outcome of the study will be the change in LM measured by dual-energy X-ray absorptiometry (Hologic fan-beam bone densitometer Discovery QDR series). The changes in lower and upper body LM are analysed separately because of differences in androgen sensitivity in leg muscles compared with neck, chest and shoulder muscles. ${ }^{62}$ Due to very similar results but greater participant comfort ${ }^{62}$ we decided to use The National Health and Nutrition Examination Survey (NHANES) protocol, which required the participant to be positioned in a supine position in the middle of the densitometry table with head straight, space between the arms and torso, palms flat on the table and feet together secured by a strap. The systematic review of Shiel $e t a l^{63}$ showed a strong level of agreement as illustrated by high intraclass correlation coefficients (ICC's) and concordance correlation coefficients (CCC's) between the Nana and NHANES positioning protocols, however systematic bias within limit of agreement plot and a large difference in 95\% confidence limits indicates that the protocols should not be interchanged when assessing an individual.

\section{Secondary outcome measures Body composition}

Other body composition parameters (fat mass, total body mass) will be measured at the same time so also the protocol is the same as with the primary outcome. The height will be measured by stadiometer and waist circumference will be measured by stretch-resistant tape that provides a constant $100 \mathrm{~g}$ tension. The body mass index is afterwards calculated and reported.

\section{Muscle strength}

Muscle strength of lower extremities will be measured as force production during maximal voluntary contraction (MVC) isometric knee extension and isometric knee flexion knee dynamometer (ARS dynamometry, S2P Ltd, Ljubljana, Slovenia). Each of the test will be performed six times with three practise trials and three recorded trials. For the first practise trial, participants will be instructed to achieve approximately $50 \%$ of the maximum, with 20 $\mathrm{s}$ rest period. The second and third practise trial will be performed at $80 \%$ of the maximum with $20 \mathrm{~s}$ rest periods. The last three trials will be performed with maximal voluntary effort and will be recorded. The best out of three will be taken for further analyses. Rest period during recorded trials will be $60 \mathrm{~s}$. During the MVC, the participants will be asked to push/pull as strong as possible and hold for $5 \mathrm{~s}$. Intrasession repeatability for MVC is the 5.7 coefficient of variation (CV) \% and 0.98 ICC. Additionally, with awareness of health issues (such as higher blood pressure) and because of a safety reasons, dynamic leg press 1RM (one repetition maximum) will be predicted from multiple repetition maximum testing. ${ }^{64}$

For assessing the muscle strength of the upper extremities, the isometric MVC handgrip strength will be measured by Camry Digital Hand Dynamometer. The participant will stand upright and hold the dynamometer in the hand next to the body, with the minimal or none flexion in the elbow joint. The base of the handle will be on the first metacarpal, while the handle should rest on the middle of the four ringers. None of the body parts will be allowed to move. The test will be performed with three practise trials. First on $50 \%$ and the others on $80 \%$ of their perceived maximum with $20 \mathrm{~s}$ ' rest period. After that, three maximum trials with rest period of $60 \mathrm{~s}$ will be recorded and the best out of three will be taken for further analyses. The participants will be encouraged to give their maximum effort. The participant will squeeze the dynamometer for $5 \mathrm{~s}$. After the test with dominant hand, the test will be performed for non-dominant hand. 


\section{Cardiorespiratory fitness}

Cardiorespiratory fitness will be measured by the single stage treadmill walking test, ${ }^{65}$ where the participants will be asked to walk on Pro Treadmill (Woodway, USA). During the walking test, participants will wear same shoes they will use during the whole intervention. The speed during the test can be changed if needed. The procedure will be performed once and heartbeat will be tracked by heart rate monitor attached on the chest. $\mathrm{VO}_{2} \max$ (the maximum rate of oxygen consumption) will be calculated according the literature. ${ }^{65}$

A $10 \mathrm{~m}$ preferred walk-speed and $10 \mathrm{~m}$ maximum walkspeed will be measured by timing gates WITTY GATE (MicroGate, Italy). Participants will walk 10 metres and the time will be measured for the intermediate 6 metres. This allow acceleration and deceleration. The gates will be placed on 2-metre mark and 8-metre mark. The timing starts when participant cross the first mark and stop when the 8-metre mark is crossed. There will be three trials for preferred and three trials for maximum walk-speed. The outcome measure will be velocity in metres per second calculated as mean of the three trials or the best trial from the preferred and maximum walk-speed test, respectively. Participants will be asked to perform at preferred walking speed first followed and then at the fastest walking speed possible.

\section{Psychosocial functioning}

The general health status will be measured by The Short Form (36) Health Survey patient-reported survey of patient health (SF-36). In addition, clinically investigating the health-related quality of life symptoms of ageing men are measured by Ageing Males' Symptom (AMS) Scale. The AMS scale had internal consistency $(\alpha=0.89(95 \%$ CI 0.88 to 0.90$)$ ); the mean alpha estimates across the AMS subscales ranged from 0.79 to 0.82 . The AMS scale also had good test-retest reliability $(r=0.85(95 \%$ CI 0.82 to 0.88$)$ ); the test-retest reliability coefficients of the AMS subscales ranged from 0.76 to $0.83 .{ }^{66}$ AMS is a standardised scale according to psychometric norms. Most of the currently available language versions were translated following international standards for linguistic and cultural translation of quality of life scales. ${ }^{67}$

\section{Serological outcomes}

Fasting morning venous blood will be taken after overnight (10 hours) fasting and $15 \mathrm{~min}$ rest from cubital vein from 8:00 am to 10:00 $\mathrm{am}^{68}$ into closed system collection tubes containing beads coated with a clotting activator and polyacryl ester-gel (Sarstedt AG \& Co, Germany). The blood will be centrifuged ( $3000 \mathrm{~g}, 4^{\circ} \mathrm{C}, 10 \mathrm{~min}$ ) immediately after sampling to obtain EDTA plasma or they will be centrifuged $\left(3000 \mathrm{~g}, 4^{\circ} \mathrm{C}, 20 \mathrm{~min}\right)$ after $30 \mathrm{~min}$ at room temperature (RT), to obtain serum. The haematological and biochemical parameters analysed immediately will be haemoglobin, haematocrit, leucocytes, thrombocytes, glucose, urea, sodium, potassium, calcium, alanineaminotransferase, total cholesterol, low-density lipoprotein cholesterol, high-density lipoprotein cholesterol, triglyceride, testosterone, oestrogen, luteinizing hormone, follicle-stimulating hormone, SHBG, albumin, bilirubin, total protein, c-reactive protein, insulin and PSA. Plasma and serum aliquots $(500 \mathrm{ul})$ will be stored at $-20^{\circ} \mathrm{C}$ (analysis within 6 months) and at $-80^{\circ} \mathrm{C}$ for the long-term storage. Bioactive molecules (myokines, exerkines, released from skeletal muscle and/or other tissues) which could be associated with the adaptive response to exercise in all patients will be quantified.

\section{Muscle cellular outcomes}

Muscle biopsies will be obtained from approximately $80 \%$ of the subjects included in the study. Subjects not willing to undergo biopsy are still eligible for trial participation. With the subject in a supine position, a $5 \mathrm{~mm}$ muscle biopsy cannula (Bergstrom-Stille, Sweden) with manual suction is used to obtain muscle samples (200 mg), under local anaesthesia (lidocaine 2\%). Before the intervention, the biopsy will be obtained from the mid-section of the right musculus vastus lateralis, and after the intervention the biopsy will be obtained $3 \mathrm{~cm}$ proximal to the pre-intervention biopsy.

\section{Muscle fibre size and regulators of muscle fibre size}

Muscle fibre size, measured as muscle fibre cross-sectional area, represents the primary muscle cellular outcome. Secondary muscle cellular outcomes reflecting regulators of muscle fibre size are (a) number of myonuclei per muscle fibre, (b) number of satellite cells per muscle fibre, (c) number of satellite cells and myonuclei positive for androgen receptors and (d) proteins involved in muscle protein degradation (muscle breakdown). The number of satellite cells will be quantified on frozen muscle cross sections with a immunohistochemical protocol as described in Bjørnsen et al. ${ }^{69}$ (Pax7 +Laminin + DAPI).

Muscle fibre cross-sectional area and regulators of muscle fibre size are analysed by immunohistochemistry on cross sections of muscle biopsies and by western blots and ELISA in muscle homogenate.

Muscle fibre cross-sectional area is measured by cutting transverse serial sections of the muscle biopsy $(8 \mu \mathrm{m}$ thick) with a cryostat microtome (Microm, Germany) at $-22^{\circ} \mathrm{C}$ and mounted on glass slides. Serial sections are immunohistochemically stained for fibre types (type I and type II) (used to measure muscle fibre cross-sectional area), number of satellite cells, number of myonuclei and number of satellite cells and myonuclei positive for androgen receptors. Muscle fibre cross-sectional area is measured for the different fibre types separately.

\section{Statistical analysis}

Normality of the data distribution will be assessed by comparing histogram of the sample data to a normal probability curve and outliers will be identified as values distant for more than $3 \sigma$ from the average. Normality will be further tested with Kolmogorov-Smirnov test if needed. 
Differences between normally distributed variables will be evaluated by the analysis of variance with repeated measures and Bonferroni post-hoc test, differences between pre-training and post-training values of the specific subpopulation will be evaluated with a paired Student's t-test. Non-normally distributed variables will be $\log$ transformed. Variables that could not be $\log$ transformed to normal distribution will be tested with non-parametric tests (Mann-Whitney test and Wilcoxon rank test).

Cohen's $d$ will be used to calculate effect size, represented by ' $\mathrm{d}$ ' and interpreted as $<0.2$ is a small, 0.2 to 0.8 is a moderate and $>0.8$ is a large effect size.

For studying the relationships between the various outcomes, the Pearson or Spearman correlation tests will be used.

All statistics were performed using a statistical software Statistical Package for the Social Sciences (SPSS) 21.0 (IBM Inc, Armonk, New York, USA) and $\mathrm{p}$ values $<0.05$ will be considered significant. Data will be presented as means and SD. Missing endpoint data will disqualify patient from the endpoint analysis. Missing single value, of training progression records will be replaced by the last observed value.

\section{Background variables}

Information about medical situation as time points for treatment and stage of symptoms are collected from the medical record. Past illnesses and other medical problems are also reported in the questionnaire.

\section{Patients and public involvement}

Patients (study participants) will be informed about the individual results of the baseline examination as well as on the primary and secondary outcomes of the study, in a form of individual consultation with a research team member.

Patients or public (patient organisations) were not involved in the development of the research question or study design. However, they will be asked to help with recruitment, and will also be involved in the conduct of the study with the power to shape (individualise) the training intervention according to individual preferences, prior experiences and medical conditions. Moreover, they will be involved in individualising the follow-up intervention protocol, shaping thus the long-term exercise programme to increase its sustainability.

\section{Sample size}

The pre-existing data from our previous 12 week exercise intervention study related to fat $(5.6 \%$ decrease $\mathrm{p}=0.002)$ $\&$ lean body mass $(1.8 \%$ increase, $\mathrm{p}=0.047$, dual-energy X-ray absorptiometry) and that of maximal voluntary contraction force measured on linear leg-press $(31 \%$ increase, $\mathrm{p}<0.0001,1 \mathrm{RM})$. Lean body mass was used to determine sample size for the population of the designed intervention study. The Type I error probability was set at 0.05 and the power to 0.90 and 0.95 . Results indicate that 22 patients per group will be sufficient to detect exercise intervention related change of $1,06 \pm 1,56 \mathrm{~kg}$ (average $\pm \mathrm{SD}$ ) of lean body mass at the power of 0.90 , accounting for the $10 \%$ patients drop-out.

\section{Ethics and dissemination}

All the participants will be fully informed on the study protocol risks and benefits and will provide the written informed consent prior to entering the study. Participation in the trial is fully voluntary. Inability to comply with the study protocol will not affect the healthcare. Data will be stored and handled anonymously using the coding system complying with the General Data Protection Regulation 2016/679. All unexpected, serious adverse events will be reported to the study sponsor as well as to the relevant health insurance company within 7 days. The findings of this trial will be published in peer-review journals, scientific conferences with main audience of healthcare professionals, healthcare providers, but also patients and their families.

\section{Author affiliations}

${ }^{1}$ Deparment of Biological and Medical Sciences, Faculty of Physical Education and Sports, Comenius University in Bratislava, Bratislava, Slovakia

${ }^{2}$ Obesity Section, Laboratory of Diabetes and Metabolic Disease, Institute of Experimental Endocrinology, Slovak Academy of Sciences, Bratislava, Slovakia ${ }^{3}$ Institute of Pathophysiology, Faculty of Medicine, Comenius University in Bratislava, Bratislava, Slovakia

${ }^{4}$ Biomedical Research Center, Slovak Academy of Sciences, Bratislava, Slovakia ${ }^{5} 5$ th Department of Internal Medicine, Faculty of Medicine, Comenius University in Bratislava, University Hospital, Bratislava, Slovakia

${ }^{6}$ Department of Urology, University Hospital - Petrzalka, Bratislava, Slovakia ${ }^{7}$ Department of Urology, Slovak Medical University Bratislava, Bratislava, Slovakia ${ }^{8}$ Department of Physical Performance, Norwegian School of Sport Sciences, Oslo, Norway

Contributors MK, JC, TR, MS participated in the study design and drafted the manuscript, GB participated in the development of the intervention protocol, TR, $\mathrm{BU}$ and $\mathrm{JU}$ designed protocol for biological sample collection and processing, and will participate in biological material sampling \& analyses. MP, ZK, JP, BK and PB provide access to patients. MS, JC, JU and MK performed data analysis. All authors contributed to and approved the present manuscript.

Funding The study is funded by the Scientific Grant Agency of the Ministry of Education, Science, Research and Sport of the Slovak Republic and of the Slovak Academy of Sciences (VEGA) no. 1/0714/16.

Competing interests None declared.

Patient consent for publication Not required.

Ethics approval This trial was approved by Ethics Committee of the University Hospital in Bratislava, Slovakia (ref. trial number: 127/2017).

Provenance and peer review Not commissioned; externally peer reviewed.

Open access This is an open access article distributed in accordance with the Creative Commons Attribution Non Commercial (CC BY-NC 4.0) license, which permits others to distribute, remix, adapt, build upon this work non-commercially, and license their derivative works on different terms, provided the original work is properly cited, appropriate credit is given, any changes made indicated, and the use is non-commercial. See: http://creativecommons.org/licenses/by-nc/4.0/.

\section{REFERENCES}

1. Florini JR. Effects of testosterone on qualitative pattern of protein synthesis in skeletal muscle. Biochemistry 1970;9:909-12.

2. Vingren JL, Kraemer WJ, Ratamess NA, et al. Testosterone physiology in resistance exercise and training: the up-stream regulatory elements. Sports Med 2010;40:1037-53. 
3. Dandona P, Rosenberg MT. A practical guide to male hypogonadism in the primary care setting. Int J Clin Pract 2010;64:682-96.

4. Kaufman JM, Vermeulen A. Declining gonadal function in elderly men. Baillière's Clinical Endocrinology and Metabolism 1997;11:289-309.

5. Tenover JS. Declining testicular function in aging men. Int $\mathrm{J}$ Impot Res 2003;15:S3-S8.

6. Feldman HA, Longcope C, Derby CA, et al. Age trends in the level of serum testosterone and other hormones in middle-aged men: longitudinal results from the Massachusetts male aging study. J Clin Endocrinol Metab 2002;87:589-98.

7. Wang C, Nieschlag E, Swerdloff R, et al. Investigation, treatment and monitoring of late-onset hypogonadism in males: Isa, ISSAM, EAU, EAA and ASA recommendations. Eur J Endocrinol 2008;159:507-14.

8. Bhasin S, Cunningham GR, Hayes FJ, et al. Testosterone therapy in adult men with androgen deficiency syndromes: an endocrine Society clinical practice guideline. J Clin Endocrinol Metab 2006;91:1995-2010.

9. Petak SM, Nankin HR, Spark RF, et al. American association of clinical endocrinologists medical guidelines for clinical practice for the evaluation and treatment of hypogonadism in adult male patients - 2002 update. Endocr Pract 2002;8:440-56.

10. Seftel A. Male hypogonadism. Part II: etiology, pathophysiology, and diagnosis. Int J Impot Res 2006;18:223-8.

11. Carnegie C. Diagnosis of hypogonadism: clinical assessments and laboratory tests. Rev Urol 2004;6 Suppl 6(suppl 6):S3-S8.

12. Collier CP, Clark AF, Bain J, et al. Functional testosterone: biochemical assessment of hypogonadism in men--report from a multidisciplinary workshop hosted by the Ontario Society of Clinical Chemists. Aging Male 2007;10:211-6.

13. Arver S, Lehtihet M. Current guidelines for the diagnosis of testosterone deficiency. Front Horm Res 2009;37:5-20.

14. Morales A, Heaton JP, Carson CC. Andropause: a misnomer for a true clinical entity. J Urol 2000;163:705-12.

15. Tremblay RR, Gagné J-M. Can we get away from serum total testosterone in the diagnosis of andropause? Aging Male 2005;8:147-50.

16. Shea JL, Wong P-Y, Chen Y, et al. Free testosterone: clinical utility and important analytical aspects of measurement. Adv Clin Chem 2014:63:59-84.

17. Smith MR, Finkelstein JS, McGovern FJ, et al. Changes in body composition during androgen deprivation therapy for prostate cancer. J Clin Endocrinol Metab 2002;87:599-603.

18. Michael H, Härkönen $\mathrm{PL}$, Väänänen $\mathrm{HK}$, et al. Estrogen and testosterone use different cellular pathways to inhibit osteoclastogenesis and bone resorption. J Bone Miner Res 2005;20:2224-32.

19. Hak AE, Witteman JCM, de Jong FH, et al. Low levels of endogenous androgens increase the risk of atherosclerosis in elderly men: the Rotterdam study. J Clin Endocrinol Metab 2002;87:3632-9.

20. Allan CA, McLachlan RI. Age-Related changes in testosterone and the role of replacement therapy in older men. Clin Endocrinol 2004;60:653-70.

21. Fink JE, Hackney AC, Matsumoto M, et al. Mobility and biomechanical functions in the aging male: testosterone and the locomotive syndrome. The Aging Male 2018;100:1-8.

22. Mäkinen JI, Perheentupa A, Irjala K, et al. Endogenous testosterone and serum lipids in middle-aged men. Atherosclerosis 2008;197:688-93.

23. Schwarz ER, Willix RD. Impact of a physician-supervised exercisenutrition program with testosterone substitution in partial androgendeficient middle-aged obese men. J Geriatr Cardiol 2011;8:201-6.

24. Grossmann M, Thomas MC, Panagiotopoulos S, et al. Low testosterone levels are common and associated with insulin resistance in men with diabetes. The Journal of Clinical Endocrinology \& Metabolism 2008;93:1834-40.

25. Ding EL, Song Y, Malik VS, et al. Sex differences of endogenous sex hormones and risk of type 2 diabetes: a systematic review and metaanalysis. JAMA 2006;295:1288-99.

26. Rosario ER, Chang L, Stanczyk FZ, et al. Age-Related testosterone depletion and the development of Alzheimer disease. JAMA 2004;292:1431-2.

27. Moffat SD, Zonderman AB, Metter EJ, et al. Longitudinal assessment of serum free testosterone concentration predicts memory performance and cognitive status in elderly men. J Clin Endocrinol Metab 2002;87:5001-7.

28. Moffat SD, Zonderman AB, Metter EJ, et al. Free testosterone and risk for Alzheimer disease in older men. Neurology 2004;62:188-93.

29. Wang C, Swerdloff RS, Iranmanesh A, et al. Transdermal testosterone gel improves sexual function, mood, muscle strength, and body composition parameters in hypogonadal men. J Clin Endocrinol Metab 2000;85:2839-53.

30. Page ST, Amory JK, Bowman FD, et al. Exogenous testosterone (T) alone or with finasteride increases physical performance, grip strength, and lean body mass in older men with low serum T. J Clin Endocrinol Metab 2005;90:1502-10.

31. Kapoor D, Aldred H, Clark S, et al. Clinical and biochemical assessment of hypogonadism in men with type 2 diabetes: correlations with bioavailable testosterone and visceral adiposity. Diabetes Care 2007;30:911-7

32. Borst SE, Mulligan T. Testosterone replacement therapy for older men. Clinical Interventions in Aging 200;2:561-6.

33. Nair KS, Rizza RA, O'Brien P, et al. DHEA in elderly women and DHEA or testosterone in elderly men. $N$ Engl J Med 2006;355:1647-59.

34. Brill KT, Weltman AL, Gentili A, et al. Single and combined effects of growth hormone and testosterone administration on measures of body composition, physical performance, mood, sexual function, bone turnover, and muscle gene expression in healthy older men. Clin Endocrinol Metab 2002;87:5649-57.

35. Clague JE, Wu FC, Horan MA. Difficulties in measuring the effect of testosterone replacement therapy on muscle function in older men. Int J Androl 1999;22:261-5.

36. Kenny AM, Kleppinger A, Annis K, et al. Effects of transdermal testosterone on bone and muscle in older men with low bioavailable testosterone levels, low bone mass, and physical frailty. J Am Geriatr Soc 2010:58:1134-43.

37. Snyder PJ, Peachey H, Hannoush P, et al. Effect of testosterone treatment on body composition and muscle strength in men over 65 years of age. J Clin Endocrinol Metab 1999;84:2647-53.

38. Zitzmann M, Nieschlag E. Androgen receptor gene CAG repeat length and body mass index modulate the safety of long-term intramuscular testosterone undecanoate therapy in hypogonadal men. J Clin Endocrinol Metab 2007;92:3844-53.

39. Lašaite L, Čeponis J, Preikša RT, et al. Effects of two-year testosterone replacement therapy on cognition, emotions and quality of life in young and middle-aged hypogonadal men. Andrologia 2017;49:e12633.

40. Hildreth KL, Barry DW, Moreau KL, et al. Effects of testosterone and progressive resistance exercise in healthy, highly functioning older men with low-normal testosterone levels. J Clin Endocrinol Metab 2013;98:1891-900.

41. Permpongkosol S, Khupulsup K, Leelaphiwat S, et al. Effects of 8-year treatment of long-acting testosterone Undecanoate on metabolic parameters, urinary symptoms, bone mineral density, and sexual function in men with late-onset hypogonadism. J Sex Med 2016;13:1199-211.

42. Bhasin S, Buckwalter JG. Testosterone supplementation in older men: a rational idea whose time has not yet come. J Androl 2001;22:718-31.

43. Barrett-Connor E, Goodman-Gruen D, Patay B. Endogenous sex hormones and cognitive function in older men. J Clin Endocrinol Metab 1999;84:3681-5.

44. Azad N, Pitale S, Barnes WE, et al. Testosterone treatment enhances regional brain perfusion in hypogonadal men. J Clin Endocrinol Metab 2003;88:3064-8.

45. Mclntyre RS, Mancini D, Eisfeld BS, et al. Calculated bioavailable testosterone levels and depression in middle-aged men. Psychoneuroendocrinology 2006;31:1029-35.

46. Kang D-Y, Li H-J. The effect of testosterone replacement therapy on prostate-specific antigen (PSA) levels in men being treated for hypogonadism: a systematic review and meta-analysis. Medicine 2015;94:e410.

47. Loeb S, Folkvaljon Y, Damber J-E, et al. Testosterone replacement therapy and risk of favorable and aggressive prostate cancer. J Clin Oncol 2017;35:1430-6.

48. Boyle P, Koechlin A, Bota M, et al. Endogenous and exogenous testosterone and the risk of prostate cancer and increased prostate-specific antigen (PSA) level: a meta-analysis. BJU Int 2016;118:731-41.

49. Cui $\mathrm{Y}$, Zong $\mathrm{H}$, Yan $\mathrm{H}$, et al. The effect of testosterone replacement therapy on prostate cancer: a systematic review and meta-analysis. Prostate Cancer Prostatic Dis 2014;17:132-43.

50. Matsumoto AM. Andropause: clinical implications of the decline in serum testosterone levels with aging in men. J Gerontol A Biol Sci Med Sci 2002;57:M76-M99.

51. Bhasin S, Woodhouse L, Casaburi R, et al. Older men are as responsive as young men to the anabolic effects of graded doses of testosterone on the skeletal muscle. J Clin Endocrinol Metab 2005;90:678-88. 
52. Rhoden EL, Morgentaler A. Risks of testosterone-replacement therapy and recommendations for monitoring. $N$ Engl $J$ Med 2004;350:482-92.

53. Crewther B, Keogh J, Cronin J, et al. Possible stimuli for strength and power adaptation: acute hormonal responses. Sports Med 2006;36:215-38.

54. Kraemer WJ, Marchitelli L, Gordon SE, et al. Hormonal and growth factor responses to heavy resistance exercise protocols. J Appl Physiol 1990;69:1442-50.

55. Newton RU, Taaffe DR, Spry N, et al. Can exercise ameliorate treatment toxicity during the initial phase of testosterone deprivation in prostate cancer patients? is this more effective than delayed rehabilitation? BMC Cancer 2012;12:432.

56. Gardner JR, Livingston PM, Fraser SF. Effects of exercise on treatment-related adverse effects for patients with prostate cancer receiving androgen-deprivation therapy: a systematic review. J Clin Oncol 2014;32:335-46.

57. Fink J, Schoenfeld BJ, Nakazato K. The role of hormones in muscle hypertrophy. Phys Sportsmed 2018;46:129-34.

58. Glintborg D, Christensen LL, Kvorning T, et al. Differential effects of strength training and testosterone treatment on soluble CD36 in aging men: possible relation to changes in body composition. Scand J Clin Lab Invest 2015;75:659-66.

59. Cho D-Y, Yeo JK, Cho SI, et al. Exercise improves the effects of testosterone replacement therapy and the durability of response after cessation of treatment: a pilot randomized controlled trial. Asian $J$ Androl 2017;19:602-7.
60. Segal RJ, Reid RD, Courneya KS, et al. Resistance exercise in men receiving androgen deprivation therapy for prostate cancer. JCO 2003;21:1653-9.

61. Eliassen W, Saeterbakken AH, van den Tillaar R. Comparison of bilateral and unilateral squat exercises on barbell kinematics and muscle activation. Int J Sports Phys Ther 2018;13:871-81.

62. Kadi F. Adaptation of human skeletal muscle to training and anabolic steroids. Acta Physiol Scand Suppl 2000;646:1-52.

63. Shiel F, Persson C, Furness J, et al. Dual energy X-ray absorptiometry positioning protocols in assessing body composition: a systematic review of the literature. J Sci Med Sport 2018;21:1038-44.

64. Reynolds JM, Gordon TJ, Robergs RA. Prediction of one repetition maximum strength from multiple repetition maximum testing and anthropometry. J Strength Cond Res 2006;20:584-92.

65. Ebbeling CB, Ward A, Puleo EM, et al. Development of a singlestage submaximal treadmill walking test. Med Sci Sports Exerc 1991;23:966-73.

66. Lee C-P, Chiu Y-W, Chu C-L, et al. A reliability generalization metaanalysis of coefficient alpha and test-retest coefficient for the aging males' symptoms (AMS) scale. Aging Male 2016;19:244-53.

67. Heinemann LAJ, Saad F, Zimmermann T, et al. The aging males' symptoms (ams) scale: update and compilation of international versions. Health Qual Life Outcomes 2003:1:15.

68. González-Sales M, Barrière O, Tremblay P-O, et al. Modeling testosterone circadian rhythm in hypogonadal males: effect of age and circannual variations. Aaps J 2016;18:217-27.

69. Bjørnsen T, Wernbom M, Kirketeig A, et al. Type 1 muscle fiber hypertrophy after blood flow-restricted training in Powerlifters. Med Sci Sports Exerc 2019;51:288-98. 